\title{
Análisis de emociones referidas por estudiantes universitarios ante distintas estrategias didácticas utilizadas durante su formación profesional
}

Analysis of emotions referred by university students

to different teaching strategies used during

their professional training

\author{
Jorge L. Olivares ${ }^{1}$ \\ Fany Arrese ${ }^{2}$ \\ Marina Villarreal ${ }^{3}$ \\ María Graciela Di Franco 4 \\ Adriana Lozano ${ }^{5}$ \\ Valeria Alfageme ${ }^{6}$ \\ Gisela N. Vincet ${ }^{7}$ \\ Noelia Damm ${ }^{8}$ \\ Mabel Carassay ${ }^{9}$
}

\footnotetext{
${ }^{1}$ Profesor Universitario. Doctor en Medicina. Investigador Categoría I del Sistema de categorización de Docentes Investigadores Universitarios del Ministerio de Educación, Cultura, Ciencia y Tecnología de la Nación. Docente de la Universidad Nacional de La Pampa, Argentina.

2 Profesora en Física. Docente de la Universidad Nacional de La Pampa, Argentina.

${ }^{3}$ Médica Pediatra. Magíster en Ciencia, Tecnología y Sociedad. Docente de la Universidad Nacional de La Pampa, Argentina.

${ }^{4}$ Magíster en Evaluación. Docente de la Universidad Nacional de La Pampa, Argentina.

${ }^{5}$ Licenciada en Psicopedagogía. Docente de la Universidad Nacional de La Pampa, Argentina.

${ }^{6}$ Profesora en Biología. Docente de la Universidad Nacional de La Pampa, Argentina.

7 Profesora en Química. Docente de nivel medio. Santa Rosa, La Pampa, Argentina.

${ }^{8}$ Estudiante del Profesorado en Ciencias Biológicas en la Universidad Nacional de La Pampa, Argentina.

9 Médica. Profesora en Biología. Docente de la Universidad Nacional de La Pampa, Argentina.

Correo electrónico de contacto: marvillarreal@cpenet.com.ar
}

Diälagos Pedagágicas. ISSN en línea: 2524-9274.

Año XVII, No 34, octubre 2019 - marzo 2020. Pág. 83-97.

DOI: http://dx.doi.org/10.22529/dp.2019.17(34)05 / Recibido: 13-08-2019 / Aprobado: 15-10-2019.

cc)(1) () $\odot$ Artículo publicado bajo Licencia Creative Commons Atribución-NoComercial-SinDerivar. (C) Un No (c) Universidad Católica de Córdoba. 
Resumen: En el espacio áulico universitario, ha prevalecido la enseñanza de contenidos disciplinares con escasa consideración de las emociones generadas. Nos propusimos identificar, en estudiantes del Profesorado en Ciencias Biológicas, cuáles son las emociones que les generan las distintas estrategias pedagógicas propuestas en dos asignaturas correlativas referidas a salud humana y analizar las respuestas en un espacio de diálogo. Se aplicó una encuesta al finalizar cada asignatura, en la que se listaban las emociones clasificadas en positivas y negativas.

Los estudiantes refirieron una mayor frecuencia de emociones positivas ante las clases dictadas por el docente. Las actividades que implicaron más participación de los estudiantes generaron el mayor número de emociones negativas. Estos futuros profesores de nivel medio refirieron que nunca habían sido consultados ni habían analizado sus emociones con anterioridad. La mayoría valoró las estrategias didácticas utilizadas ya que consideran que los colocaron en un rol participativo y que favorecieron su formación profesional.

Palabras clave: emoción, educación científica, formación de profesores, universidad.

Abstract: Within the academic space, the teaching of disciplinary content has prevailed with poor consideration of the generated emotions. We set out to identify, in the Biological Sciences students, which were the emotions generated by the different pedagogical strategies proposed in two correlative subjects related to human health and analyze the answers in a space for dialogue. A survey was applied at the end of each subject, where emotions were classified as positive and negative.

The students reported a higher frequency of positive emotions in response to classes taught by the teacher. The activities that involved more student participation generated the greatest number of negative emotions. These future middle-level teachers stated they had never been consulted or analyzed their emotions before. The majority of the students valued the didactic strategies used and they considered they were placed in a participative role, which favored their professional training.

Keywords: emotion, science education, teacher training, university.

\section{Introducción}

En la formación docente de los estudiantes del Profesorado en Ciencias Biológicas en nuestra universidad, ha prevalecido la enseñanza de contenidos disciplinares, con escasa consideración de las emociones que se generan en el proceso de enseñanza y aprendizaje.

Desde el constructivismo, marco teórico destacado en las últimas décadas en la didáctica de las ciencias, el cambio conceptual comienza a incluir la consideración de las emociones como complemento del cambio cognitivo. Hargreaves (1996, 1998) plantea que las emociones están en el corazón de la enseñanza y el apren- 
dizaje. En realidad, afirma Hernández (2002), lo cognitivo configura lo afectivo y lo afectivo, lo cognitivo.

En el plano pedagógico, las emociones son reconocidas como el otro aspecto del conocimiento didáctico del contenido (Mellado, Borrachero, Brígido, Melo, Dávila, Cañada et al., 2014), porque facilitarán que los sujetos de aprendizaje sientan confianza cuando sean interpelados ante nuevos objetos de estudio y puedan socialmente contextualizarlos para generar un aprendizaje relevante y ético (Kolb \& Kolb, 2001; Camilloni, 2015; Camps, 1993).

Es importante aclarar que no nos referimos a las emociones como sinónimos de sentimientos. Aunque hay diferentes conceptualizaciones, para nuestro estudio, diferenciamos los términos y consideramos a las emociones como percepciones subjetivas que generan respuestas psicobiológicas inconscientes, a diferencia de los sentimientos, que son respuestas conscientes de determinadas emociones ante distintos componentes cognitivos y subjetivos. Las emociones también se diferencian de los sentimientos en que son de corta duración y más intensas y en que son producto de respuestas automáticas de nuestro cuerpo (Damasio, 2010). De esta manera, en el ámbito educativo, una emoción se genera ante diferentes estímulos externos e internos que desencadenan diferentes respuestas biológicas y conductuales, las cuales van a generar sentimientos particulares a partir de las experiencias o antecedentes de vida del sujeto expuesto e involucran sus aspectos sociales y culturales (Bisquerra, 2000; Damasio, 2010).

Desde una perspectiva sociocultural de la didáctica de las ciencias, Tobin (2010, p. 303) señala que -en el aprendizaje de las ciencias- "las emociones actúan como un pegamento social que interconecta intereses y acciones individuales y colectivas". Los estados emocionales positivos favorecen el aprendizaje de las ciencias y el compromiso de los estudiantes como aprendices activos, mientras que los negativos limitan la capacidad de aprender (Vázquez \& Manassero, 2007). Por lo tanto, el espacio de formación universitario podría ser fundante para que los futuros profesores efectúen la posterior transposición didáctica en el plano disciplinar que enseñarán y, también, para la formación de habilidades socioemocionales (Fragoso Luzuriaga, 2018).

Vázquez y Manassero (2007) destacan que la investigación sobre las emociones implica tanto analizar aspectos generales como específicos relacionados con los contenidos disciplinares de las diferentes materias, que deben ser objeto de estudio desde las didácticas específicas, ya que las emociones también están ligadas a la enseñanza-aprendizaje de conocimientos concretos (Dolan, 2002; Garritz \& Ortega-Villar, 2013).

En este sentido, en cuanto a investigar didácticas específicas, en particular las referidas a conocimientos sobre salud humana, se ha investigado -en nuestra facultad- la influencia que puede tener en la formación de futuros profesores su propia historia emocional al identificar sus recuerdos o emociones en respuesta a materias cursadas en la escuela secundaria (Olivares \& Morales, 2013). Para Mellado et al. 2014, esos recuerdos o causas internas pueden tener más influencia en la formación profesional que las causas externas. 
El estudio que presentamos se ubica dentro de las investigaciones de las emociones, en particular, a las referidas por los estudiantes del Profesorado en Ciencias Biológicas ante distintas estrategias didácticas utilizadas en asignaturas de salud humana.

\section{Objetivo}

Nos propusimos identificar, en estudiantes universitarios del Profesorado en Ciencias Biológicas, cuáles son las emociones que les generan las distintas estrategias didácticas, compararlas al finalizar de cursar dos asignaturas correlativas que implementan dichas estrategias y analizar la información obtenida en un espacio de diálogo entre docentes y estudiantes.

\section{Metodología}

Esta investigación se realizó desde una perspectiva que involucra datos cuantitativos y cualitativos; es decir, un acercamiento mixto. Se encuentra incluido en el Proyecto de evaluación de estrategias pedagógicas PI 25 CB, Resolución 38/18 de la Facultad de Ciencias Exactas y Naturales de la Universidad Nacional de la Pampa (UNLPam). Es un trabajo de carácter exploratorio, realizado con estudiantes avanzados del Profesorado en Ciencias Biológicas, en el que se indagan las emociones que les generaron las distintas estrategias didácticas empleadas en las asignaturas correlativas Cuerpo Humano I y II durante 2018. La primera asignatura se dicta en el primer cuatrimestre de marzo a junio y la segunda, de agosto a noviembre.

La propuesta de las asignaturas que tratan contenidos de Salud Humana, Cuerpo Humano I y Cuerpo Humano II, es que los estudiantes universitarios del Profesorado en Ciencias Biológicas de la Universidad Nacional de La Pampa transiten -en su formación profesional- por distintos espacios, en los que la teoría se amalgame con la práctica disciplinar y pedagógica. Se abordan situaciones de la vida real, en las cuales el conocimiento disciplinar de la salud humana está inmerso en relaciones con factores sociales, culturales y afectivos (Vázquez \& Manassero, 2007). La intención es evitar la enseñanza lineal de conocimientos abstractos y poco relevantes para la vida extraescolar, que no permiten el disfrute durante la formación profesional (Vázquez \& Manassero, 2007).

Para lograrlo, se utilizan diversas estrategias didácticas. Desde el inicio de la cursada en marzo, se plantea que la disposición en el aula de los estudiantes sea en círculo con un frente común. Esta estrategia se utiliza para generar confianza, participación permanente y la posibilidad de un acercamiento comunicacional en la interacción docente-estudiante (Oosterheert \& Vermunt, 2001).

Las estrategias didácticas implementadas que se analizaron para el presente estudio fueron tres. Dos de ellas son utilizadas en la presentación de la teoría y una se implementa en actividades prácticas en el aula. Las estrategias teóricas fueron el dictado de clases por parte del docente y las exposiciones conjuntas 
docente-estudiante. La estrategia que se evaluó en las actividades prácticas en el aula fue el denominado aprendizaje basado en problemas (ABP).

Los dos tipos de clases teóricas son dialógicas, con permanente interacción docente-estudiante (Coll \& Sánchez, 2008). Las clases teóricas conjuntas docente-estudiante brindan -además- un espacio pedagógico donde cada estudiante puede practicar un discurso disciplinar profesional, ejercitar la oralidad y organizar los contenidos según sus propios criterios e interpretaciones, siempre con el acompañamiento, las sugerencias y la supervisión del docente de la asignatura.

En las actividades prácticas en que se utiliza el ABP, el objetivo es la aplicación de conocimientos para consolidar y generar aprendizajes relevantes (Kolb \& Kolb 2001; Camilloni, 2015). Las temáticas abordadas incluyen problemas prevalentes en nuestro medio. Para ejemplificar, en la Unidad de Sistema Hematopoyético, se aborda la problemática del requerimiento de transfusiones de sangre en la población y sus motivos, los componentes de la sangre que se utilizan con mayor frecuencia y las dificultades del banco de sangre para hacer frente a esas necesidades. Los conceptos centrales se refieren a dos problemas sanitarios y socioculturales: la alta prevalencia de siniestros de tránsito en Argentina y la necesidad de avanzar en el altruismo de la población para asistir a donar sangre voluntariamente, dada la dificultad de los bancos de sangre para afrontar esta realidad sin la colaboración masiva de la población. En el análisis de estos problemas, algún estudiante participa con los docentes para conducir u organizar las líneas de discusión con el resto de los estudiantes. Por otro lado, los temas abordados como ABP aumentan el interés por las actividades extramuros que se desarrollan posteriormente. En el caso del sistema hematopoyético, los estudiantes luego concurren al banco de sangre de un hospital zonal, que se transforma temporalmente en un espacio diferente del aula, donde pueda generarse, en los estudiantes, el aprendizaje experiencial (Kolb \& Kolb, 2001).

En los tres tipos de estrategia didáctica se estimula la intervención, se les alienta a adoptar un rol activo y a desarrollar un espíritu crítico. Son todos aspectos que se espera que reproduzcan en su futuro como profesionales docentes de nivel medio.

Para obtener la opinión de los estudiantes sobre las estrategias descriptas, se confeccionó un instrumento considerando la clasificación de las emociones evaluadas por otros autores (Magnusson, Krajcik \& Borko, 1999; Mellado et al., 2014). Se trata de una encuesta estructurada y voluntaria, en la que se plasma un listado de ocho emociones positivas y ocho negativas. Las positivas fueron alegría, confianza, felicidad, admiración, tranquilidad, satisfacción, sorpresa y diversión. Las emociones negativas que podían elegir fueron vergüenza, miedo, nerviosismo, ansiedad, preocupación, ira, asco y tristeza. Se les explicó a los estudiantes que se realizaba la encuesta para conocer qué emociones les habían generado las tres estrategias didácticas empleadas durante el cursado de las asignaturas Cuerpo Humano I y Cuerpo Humano II. Se les aclaró que no había límite en el número de emociones que podían marcar. 
La primera encuesta voluntaria se realizó al finalizar el cursado de la asignatura Cuerpo Humano I, en junio de 2018. La misma encuesta se realizó al finalizar el cursado de Cuerpo Humano II, en noviembre del mismo año, para evaluar si cambiaban las emociones hacia el final del año con la reiterada aplicación de las estrategias evaluadas. La segunda encuesta fue respondida por menos estudiantes, ya que tres de ellos cursaron solo la primera asignatura.

Con posterioridad a la obtención de la información, se generó un espacio de diálogo para que docentes y estudiantes pudieran analizar las respuestas a las encuestas.

\section{Resultados}

En 2018, trece estudiantes cursaron Cuerpo Humano I y diez cursaron Cuerpo Humano II, ambas asignaturas del Profesorado en Ciencias Biológicas de la Facultad de Ciencias Exactas y Naturales de la Universidad Nacional de La Pampa (UNLPam).

Todos ellos aceptaron responder las encuestas y participar del diálogo posterior en torno a los resultados. Ninguno tuvo dudas respecto de la comprensión de lo solicitado ni sobre el significado de cada emoción listada.

El conjunto de estos estudiantes avanzados, que se encuentran finalizando el tercer año de un profesorado de cuatro años de duración, refirieron que nunca antes se les había solicitado en su carrera expresar sus emociones respecto de ningún aspecto. Anualmente, la Secretaría Académica les solicita a los estudiantes que plasmen su opinión en el sistema informático de la facultad para evaluar el desempeño y cumplimiento de cada asignatura, pero no se incluye ningún aspecto referido a sus emociones o sentimientos.

\section{Primera encuesta (al finalizar la asignatura Cuerpo Humano I)}

Para las tres estrategias didácticas evaluadas, los estudiantes seleccionaron más emociones positivas que negativas.

Puede observarse en la Tabla 1 que la emoción positiva más frecuentemente elegida para las tres estrategias fue la confianza. El dictado de clases por el docente fue la estrategia que más confianza y tranquilidad les generó, incluso mayor que el dictado conjunto entre docente y estudiante, donde el estudiante debe asumir un rol más activo.

Respecto de la práctica evaluada, puede verse en Tabla 1, en el ítem "actividad práctica", que para el aprendizaje basado en problemas (ABP) la emoción referida con mayor frecuencia también fue la confianza, aunque se destacan la sorpresa y la satisfacción.

La estrategia didáctica que acumuló más emociones negativas fue la clase conjunta docente-estudiante, que generó vergüenza (23\%), miedo (15\%), nerviosismo (15\%) y ansiedad (7\%). La actividad que menos emociones negativas 
J. L. Olivares, F. Arrese, M. Villarreal, M. G. Di Franco, A. Lozano, V. Alfageme, G. N. Vincet, N. Damm, M. Carassay

provocó fue la práctica con ABP, que generó preocupación (15\%), nerviosismo (7\%) y ansiedad (7\%). Muy similar a la práctica de ABP fue el porcentaje de emociones negativas referidas ante las clases dialógicas dictadas por el docente, que generaron nerviosismo (23\%) y preocupación (7\%) en los estudiantes.

\section{Segunda encuesta (al finalizar la asignatura Cuerpo Humano II)}

Los estudiantes seleccionaron muchas más emociones positivas que negativas para las tres estrategias didácticas.

Tabla 1. Emociones positivas y negativas ante las tres estrategias didácticas empleadas en Cuerpo Humano I. Encuesta a trece estudiantes del Profesorado en Ciencias Biológicas, junio 2018

\begin{tabular}{|c|c|c|c|c|c|c|}
\hline \multirow{3}{*}{$\begin{array}{l}\begin{array}{c}\text { Tipos de } \\
\text { emociones }\end{array} \\
\text { Alegría }\end{array}$} & \multicolumn{4}{|c|}{ Clase dialógica } & \multirow{2}{*}{\multicolumn{2}{|c|}{\begin{tabular}{|c|} 
Actividad práctica \\
$A B P$
\end{tabular}}} \\
\hline & \multicolumn{2}{|c|}{$\begin{array}{l}\text { Dictadas por } \\
\text { docente }\end{array}$} & \multicolumn{2}{|c|}{$\begin{array}{c}\text { Dictadas por } \\
\text { docente } y \text { estudiante }\end{array}$} & & \\
\hline & 1 & $7 \%$ & 2 & $15 \%$ & 0 & - \\
\hline Confianza & 10 & $77 \%$ & 9 & $69 \%$ & 8 & $61 \%$ \\
\hline Felicidad & 1 & $7 \%$ & 1 & $7 \%$ & 0 & - \\
\hline Admiración & 1 & $7 \%$ & 1 & $7 \%$ & 0 & - \\
\hline Tranquilidad & 4 & $31 \%$ & 4 & $30 \%$ & 2 & $15 \%$ \\
\hline Satisfacción & 1 & $7 \%$ & 2 & $15 \%$ & 5 & $38 \%$ \\
\hline Sorpresa & 1 & $7 \%$ & 1 & $7 \%$ & 7 & $54 \%$ \\
\hline Diversión & 2 & $14 \%$ & 5 & $38 \%$ & 2 & $15 \%$ \\
\hline Vergüenza & 0 & - & 3 & $23 \%$ & 0 & . \\
\hline Miedo & 0 & - & 2 & $15 \%$ & 0 & - \\
\hline Nerviosismo & 3 & $23 \%$ & 2 & $15 \%$ & 1 & $7 \%$ \\
\hline Ansiedad & 0 & - & 1 & $7 \%$ & 1 & $7 \%$ \\
\hline Preocupación & 1 & $7 \%$ & 0 & - & 2 & $15 \%$ \\
\hline Ira & 0 & - & 0 & - & 0 & - \\
\hline Asco & 0 & - & 0 & - & 0 & - \\
\hline Tristeza & 0 & - & 0 & - & 0 & - \\
\hline
\end{tabular}

Nota: El número de emociones por columna para cada estrategia didáctica es mayor que el número total de estudiantes, ya que cada uno podía marcar tantas emociones como deseara. 
Puede observarse en la Tabla 2 que las clases dictadas por el docente generaron el mayor porcentaje de emociones positivas, como sucedió en la primera encuesta, en las que se destacan la confianza, la alegría y la tranquilidad.

Las emociones negativas seleccionadas en esta segunda encuesta disminuyeron marcadamente para las tres estrategias evaluadas. El mayor porcentaje de emociones negativas fueron referidas por los estudiantes para las clases conjuntas docente-estudiante: nerviosismo (20\%), vergüenza (10\%) y miedo (10\%).

Tabla 2. Emociones positivas y negativas ante las tres estrategias didácticas empleadas en Cuerpo Humano II. Encuesta a diez estudiantes del Profesorado en Ciencias Biológicas, noviembre 2018

\begin{tabular}{|c|c|c|c|c|c|c|}
\hline \multirow{3}{*}{$\begin{array}{l}\begin{array}{r}\text { Tipos de } \\
\text { emociones }\end{array} \\
\text { Alegría }\end{array}$} & \multicolumn{4}{|c|}{ Clase dialógica } & \multirow{2}{*}{\multicolumn{2}{|c|}{$\begin{array}{c}\text { Actividad práctica } \\
A B P\end{array}$}} \\
\hline & \multicolumn{2}{|c|}{$\begin{array}{l}\text { Dictadas por } \\
\text { docente }\end{array}$} & \multicolumn{2}{|c|}{$\begin{array}{c}\text { Dictadas por } \\
\text { docente } y \text { estudiante }\end{array}$} & & \\
\hline & 8 & $80 \%$ & 5 & $50 \%$ & 1 & $10 \%$ \\
\hline Confianza & 8 & $80 \%$ & 7 & $70 \%$ & 2 & $30 \%$ \\
\hline Felicidad & 3 & $30 \%$ & 4 & $40 \%$ & 1 & $10 \%$ \\
\hline Admiración & 1 & $10 \%$ & 2 & $20 \%$ & 3 & $30 \%$ \\
\hline Tranquilidad & 7 & $70 \%$ & 2 & $20 \%$ & 3 & $30 \%$ \\
\hline Satisfacción & 3 & $30 \%$ & 4 & $40 \%$ & 4 & $40 \%$ \\
\hline Sorpresa & 3 & $30 \%$ & 3 & $30 \%$ & 4 & $40 \%$ \\
\hline Diversión & 3 & $30 \%$ & 3 & $40 \%$ & 4 & $40 \%$ \\
\hline Vergüenza & 0 & - & 1 & $10 \%$ & 0 & - \\
\hline Miedo & 0 & - & 1 & $10 \%$ & 0 & - \\
\hline Nerviosismo & 1 & $10 \%$ & 2 & $20 \%$ & 0 & - \\
\hline Ansiedad & 0 & - & 0 & - & 0 & - \\
\hline Preocupación & 0 & - & 0 & - & 0 & - \\
\hline Ira & 0 & - & 0 & - & 0 & - \\
\hline Asco & 0 & - & 0 & - & 0 & - \\
\hline Tristeza & 0 & - & 0 & - & 0 & - \\
\hline
\end{tabular}

Nota: El número de emociones por columna para cada estrategia didáctica es mayor que el número total de estudiantes, ya que cada uno podía marcar tantas emociones como deseara. 


\section{Comparación entre la primera y la segunda encuesta (referidas a Cuerpo Humano II y Cuerpo Humano II, respectivamente)}

Para las tres estrategias evaluadas, en la segunda encuesta -es decir, al finalizar el cursado de Cuerpo Humano II- el número de emociones positivas elegidas por los estudiantes fue mayor, y el de emociones negativas fue menor.

Particularmente, en relación a las clases dictadas solo por el docente, pueden verse en el Gráfico 1 las diferencias en las emociones seleccionadas en las dos encuestas. Al finalizar el año, esta estrategia se mantuvo como la que más emociones positivas generó y aumentó la tranquilidad y la diversión. Asimismo, las clases dictadas por el docente motivaron el menor número de emociones negativas, las cuales disminuyeron aún más hacia fin de año. Al finalizar la asignatura Cuerpo Humano II, sólo tres estudiantes refirieron alguna emoción negativa y coincidieron en la elección de la emoción nerviosismo.

Respecto de la estrategia de clase dictada en conjunto por estudiante y docente, en la segunda encuesta, los estudiantes también seleccionaron un mayor número de emociones positivas, con un notorio aumento de la alegría. Las emociones negativas, en rasgos generales, disminuyeron, aunque aumentó el nerviosismo. En el Gráfico 2, se muestra la comparación de las emociones predominantes seleccionadas en ambas encuestas.

En relación a la estrategia de actividades prácticas que utilizan $A B P$, se observa en el Gráfico 3 que la sorpresa disminuyó, se mantuvo la satisfacción, pero se incrementaron la tranquilidad y la diversión. Los estudiantes no seleccionaron ninguna emoción negativa a fin de año para esta estrategia.

\section{Gráfico 1. Comparación de emociones positivas y negativas predominantes ante clases dictadas por el docente, según la asignatura}

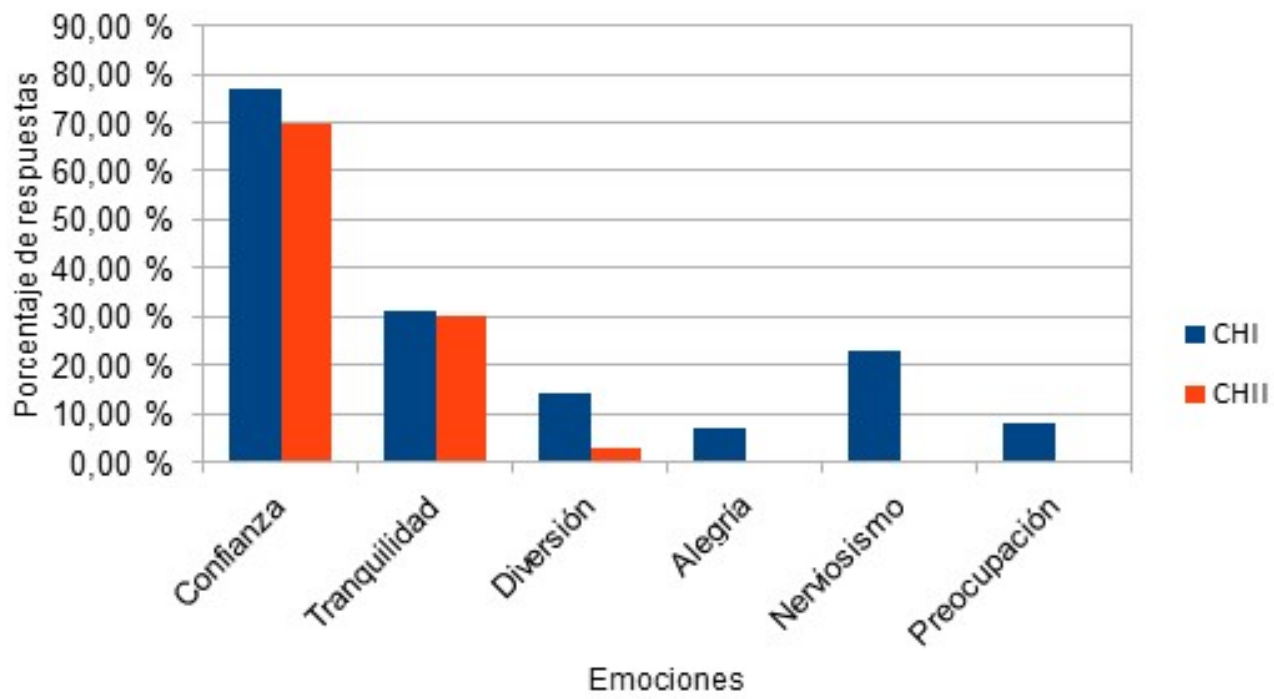




\section{Diálogo entre docentes y estudiantes sobre las encuestas realizadas}

Luego de efectuada la segunda encuesta, y habiendo finalizado el año de cursado de ambas asignaturas correlativas, se dialogó con los estudiantes sobre los resultados de las encuestas en un espacio de opinión grupal. Como se refirió previamente, todos los estudiantes manifestaron asombro por haber sido invitados a explicitar sus emociones. A ningún estudiante le resultó difícil reconocer que las estrategias pedagógicas utilizadas les generaron distintas emociones, solo les pareció totalmente inusual que se les pidiera expresarlas y reflexionar sobre ellas.

Fue mayoritaria la opinión respecto de lo dificultoso que les resultó a los estudiantes adaptarse al comenzar el año a una modalidad de clases dialógicas, con participación activa, especialmente a las clases en que el estudiante participa del dictado de clases, aunque lo hiciera en forma conjunta con un docente. Pese a ser la estrategia más similar a lo que desearían implementar en su futuro docente, fue la que motivó más emociones negativas. Lo adjudicaron principalmente al hecho de no haber tendido entrenamiento previo en pasar al frente, no solo para presentar contenido, sino para estimular el intercambio con sus compañeros desde

\section{Gráfico 2. Comparación de emociones positivas y negativas predominantes ante clases dictadas por docente y estudiante, según la asignatura}

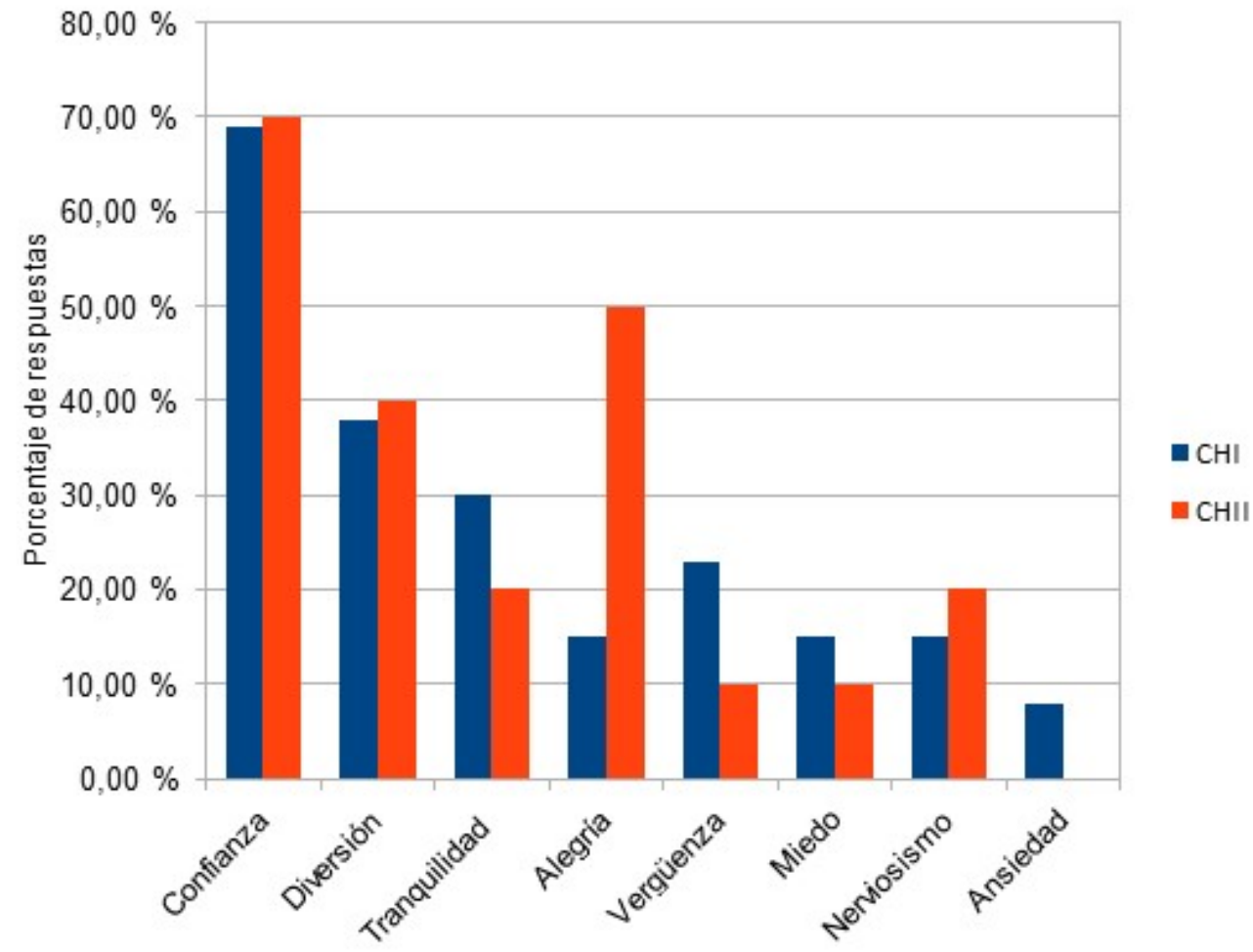


un rol docente. Destacaron, sin embargo, que el mejor escenario para practicar, con las emociones que ello implica, sin dudas, es la universidad.

Respecto de por qué pensaban que las clases a cargo exclusivamente del docente les generaron más emociones positivas y menos negativas, refirieron que fue porque se asemejaban más a las clases tradicionales a las que están acostumbrados.

La mayoría de los estudiantes valoró las estrategias didácticas utilizadas, consideraron que los colocaron en una posición de estudiante participativo y que favorecieron su formación profesional.

\section{Discusión}

En las últimas décadas, en la investigación de la enseñanza, vuelve a reconocerse que el proceso enseñanza-aprendizaje de conceptos científicos es mucho más que la suma de procesos cognitivos y que, también, está fuertemente cargado de emociones (Garritz, 2010).

Los estudiantes que participaron de este estudio exploratorio no tuvieron dudas respecto de lo que se les solicitaba, rápidamente reconocieron la existencia de diversas emociones en el proceso de enseñanza-aprendizaje de su carrera en curso y, en particular, ante las estrategias pedagógicas implementadas en las asignaturas Cuerpo Humano I y II, que abordan contenidos sobre salud humana. No

\section{Gráfico 3. Comparación de emociones positivas y negativas predominantes ante las actividades prácticas de tipo $A B P$, según la asignatura}

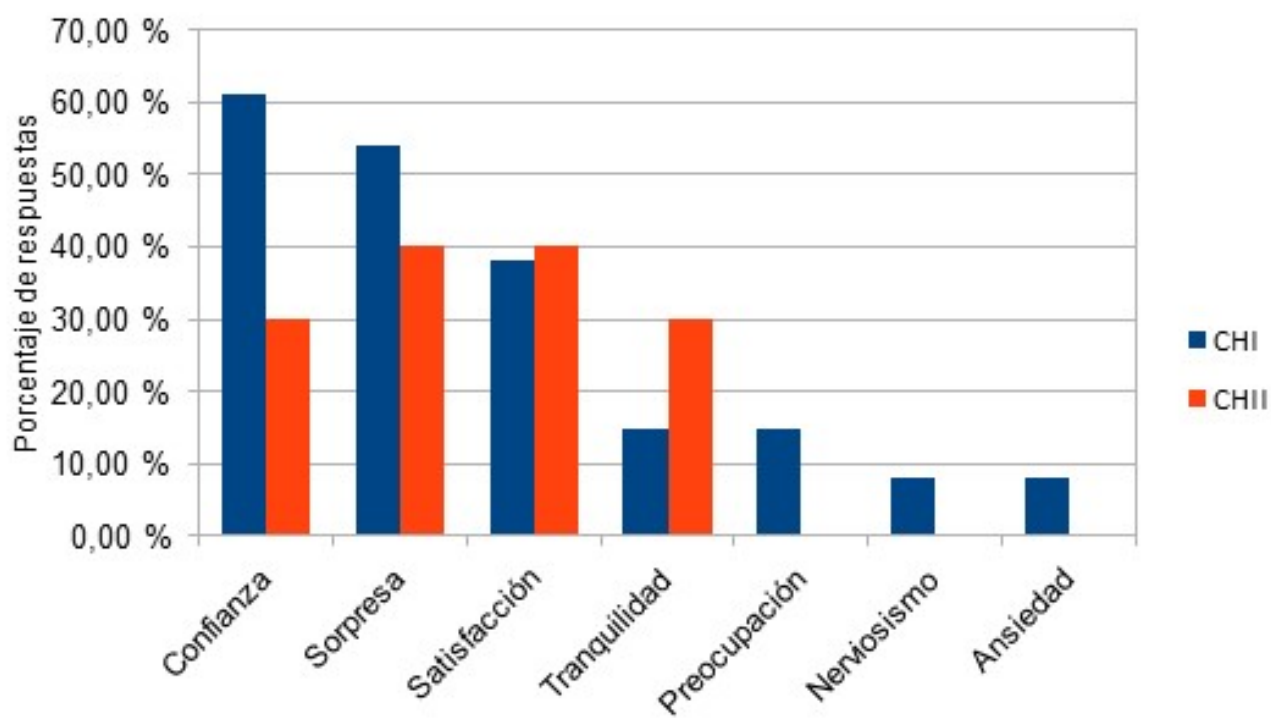


dudaron ni preguntaron sobre el significado de las emociones, pese a la diversidad de definiciones existentes en la literatura (Bisquerra \& Pérez, 2007; Manassero, 2013; Mellado et al., 2014). Por el contrario, pese al asombro ante el pedido de que explicitaran sus emociones, consideraron que sus aprendizajes, incluido el intercambio con sus pares y docentes, siempre han involucrado emociones sobre las cuales no habían reflexionado ni se les había solicitado reflexionar con anterioridad. Al respecto, Garritz (2010) ha afirmado que, tanto en escuelas como en universidades, mayormente, la ciencia está representada como un área del currículum racional, analítica y no emotiva y que los profesores de ciencias, los textos y los documentos curriculares -comúnmente- presentan imágenes de la ciencia y de los científicos que plasman una gran distancia con lo emocional.

Del análisis de la asignatura Cuerpo Humano I, al finalizar el primer cuatrimestre del año (junio), se destacó la alta frecuencia de emociones positivas generadas por las clases a cargo exclusivamente del docente. Llamativamente, en estos futuros profesores de Biología, el dictado de clases en las que algún estudiante asumía un rol docente, si bien tuvo un porcentaje alto de emociones positivas, fue la estrategia que generó el mayor porcentaje de emociones negativas.

Para Manassero (2013, p. 8), "las emociones sirven para motivar, organizar, dirigir y activar conductas, pero también pueden bloquear o interrumpir otras conductas". Se ha descripto que las primeras experiencias durante las prácticas de enseñanza en aulas del nivel medio son emocionalmente muy fuertes y pueden ser traumáticas para los futuros profesores que -en esta etapa- son especialmente vulnerables. Las emociones negativas en las primeras experiencias de enseñanza pueden fijar estrategias conductistas, dirigidas hacia el control y la supervivencia, que son muy resistentes al cambio en el futuro (Frenzel, Goetz, Stephens \& Jacob, 2011; Ritchie, Tobin, Hudson, Roth \& Mergard, 2011). Por lo tanto, la importancia de aplicar la estrategia en que el estudiante colabora con el docente en el dictado de clases dialógicas, aunque genere emociones negativas en el aula de la universidad, radica en que podría prevenir o disminuir en estos futuros profesores sus emociones negativas cuando estén frente a estudiantes de nivel medio. $Y$, tal vez, podrían disminuir el uso futuro de estrategias conductistas.

Al evaluar los resultados de las encuestas y comparar su proceso de formación entre marzo y noviembre, los estudiantes destacaron la progresiva valoración de las estrategias centradas en estimular la participación del estudiante. Reconocieron fundamentalmente que, en la medida que se utilizaron, les resultaron más importantes, especialmente como futuros profesionales de la educación. Y, a pesar de que el mayor número de emociones negativas correspondió a la estrategia de clases conjuntas estudiante-docente (aunque disminuyeron hacia el final del año), afirmaron que la participación del estudiante en el dictado de clases teóricas es una herramienta de formación muy importante.

Damasio (2010) ha señalado que lo que acarrea emociones negativas solo puede ser contrarrestado generando emociones positivas aún más fuertes. Algunos programas de formación profesional en España han comenzado a implementar programas de intervención para cambiar emociones negativas por positivas (Me- 
llado et al., 2014). Estas intervenciones podrían ser especialmente importantes para las mujeres, quienes han reportado mayor cantidad de emociones negativas que los hombres (Borrachero, 2014).

El espacio de diálogo generado luego de las encuestas permitió poner en discusión que el componente afectivo es un rasgo relevante de la actitud para formar profesionales, que su reconocimiento y abordaje facilitarán un cambio de paradigma en la formación de docentes no solo formados científicamente, sino en lo emocional, como han propuesto Vázquez y Manassero (2007).

Una debilidad de este trabajo es el bajo número de estudiantes evaluados (aunque son el total de los cursantes). Sin embargo, al momento de aplicar estrategias didácticas centradas en el alumno, de estimular su participación activa y de propiciar el diálogo, el bajo número constituyó una ventaja y una oportunidad para el acercamiento con el docente.

Finalmente, debemos destacar el acompañamiento de nuestra facultad en la aplicación y la evaluación de estas estrategias didácticas constructivistas centradas en el estudiante. Lograr el apoyo de las autoridades constituye uno de los retos para el desarrollo de la educación emocional en la universidad (Fragoso Luzuriaga, 2018). La investigación de Peme-Aranega, Mellado, De Longhi, Argañaraz y Ruiz (2008), sobre una profesora preparada para aplicar estrategias innovadoras, mostró la dificultad de que se realicen cambios didácticos y -sobre todo- que se consoliden de una forma individual y a contracorriente de la cultura del centro educativo y de las formas socialmente asumidas. En nuestro caso, se contó con el acompañamiento de las autoridades $y$, también, de colegas docentes de distintas áreas. $Y$, si consideramos las opiniones de los estudiantes que participaron de este estudio, que fueron la totalidad de los que cursaron las asignaturas Cuerpo Humano I y II, también se contó con el apoyo de los futuros profesores, conscientes de que su institución formadora es el escenario ideal para reconocer sus emociones y para reflexionar sobre ellas.

\section{Conclusiones}

Las estrategias didácticas evaluadas generaron mayores porcentajes de emociones positivas que negativas y esta tendencia se incrementó hacia fin de año, al terminar el cursado de las dos asignaturas correlativas evaluadas. La estrategia para la cual los estudiantes seleccionaron el mayor número de emociones positivas y el menor número de negativas fueron las clases dictadas por el docente. Las clases en que un estudiante colaboraba con el docente, por el contrario, generaron menos opiniones positivas y más negativas, aunque la tendencia mejoró hacia fin de año. Un espacio de diálogo permitió a los estudiantes reflexionar sobre sus elecciones y reconocer que no están acostumbrados a asumir un rol activo. La implementación y evaluación de estas tres estrategias didácticas centradas en el alumno fueron valoradas positivamente por los estudiantes, los docentes y las autoridades de la facultad. 


\section{Referencias bibliográficas}

Bisquerra, R. (2000). Educación emocional y bienestar. Barcelona: Praxis.

Bisquerra, R. \& Pérez, N. (2007). Las competencias emocionales. Educación XXI, 10, 61-82.

Borrachero, A. B., Brígido, M., Mellado, L., Costillo, E. \& Mellado, V. (2014). Emotions in prospective secondary teachers when teaching science content, distinguishing by gender. Research in Science \& Technological Education, 32, Issue 2, 182-215. Recuperado el 23 de abril de 2018, de: https://www.tandfonline.com/doi/full/ $10.1080 / 02635143.2014 .909800$ ?scroll=top\&needAccess $=$ true

Camilloni, A. (2015). Docencia, investigación y extensión: un vínculo necesario. Cuadernos de Extensión Universitaria de la UNLPam, 1(1), 11-21. Recuperado el 17 de mayo de 2019, de: http://www.unlpam.edu.ar/images/extension/ cuadernos.pdf

Camps, V. (1993). Los valores de la educación. Madrid: Alauda/Anaya.

Coll, C. \& Sánchez, E. (2008). Presentación. El análisis de la interacción alumnoprofesor: líneas de investigación. Revista de Educación, 346, 15-32.

Damasio, A. (2010). Y el cerebro creó al hombre. Barcelona: Destino.

Dolan, R. J. (2002). Emotion, Cognition, and Behavior. Science, 298, 1191-1194.

Fragoso Luzuriaga, R. (2018). Retos y herramientas generales para el desarrollo de la Inteligencia Emocional en las aulas universitarias. Praxis Educativa, 22(3), 47-55. Recuperado el 10 mayo de 2019, de: https://www.redalyc.org/ articulo.oa?id=153157080006.

Frenzel, A. C., Goetz, T., Stephens, E. J. \& Jacob, B. (2011). Antecedents and effects of teachers' emotional experiences: An integrated perspective and empirical test. En P. A. Schutz \& M. Zembylas (Eds.), Advances in teacher emotion research (pp. 129-151). Dordrecht: Springer.

Garritz, A. (2010). Pedagogical content knowledge and the affective domain of scholarship of teaching and learning. International Journal for the Scholarship of Teaching and Learning, 4(2), 1-6. Recuperado el 22 de octubre de 2018, de: https:/ /andoni.garritz.com/documentos/2013/01_GarritzAffectivePCKIJSoTL2010.pdf.

Garritz, A. \& Ortega-Villar, N. A. (2013). El aspecto afectivo en la enseñanza universitaria. Cómo cinco profesores enseñan el enlace químico en la materia condensada. En V. Mellado, L. J. Blanco, A. B. Borrachero \& J. A. Cárdenas (Eds.), Las emociones en la enseñanza y el aprendizaje de las ciencias y las matemáticas (pp. 277-304). Badajoz: UEX-DEPROFE.

Hargreaves, A. (1996). Profesorado, cultura y modernidad. Madrid: Morata.

Hargreaves, A. (1998). The emotions of teaching and educational change. En A. 
J. L. Olivares, F. Arrese, M. Villarreal, M. G. Di Franco, A. Lozano, V. Alfageme, G. N. Vincet, N. Damm, M. Carassay

Hargreaves, A. Lieberman, M. Fullan \& D. Hopkins (Eds.), International handbook of educational change (pp. 558-575). Dordrecht/ Boston/ London: Kluwer Academic Publishers.

Hernández, P. (2002). Los moldes de la mente. Más allá de la inteligencia emocional. Tenerife: Tafor.

Kolb, D. \& Kolb, A. (2001). Experiential Learning Theory Bibliography 1971-2001. Boston: McBer and Co.

Magnusson, S., Krajcik, J. \& Borko, H. (1999). Nature, sources, and development of the PCK for science teaching. En J. Gess-Newsome \& N. G. Lederman (Eds.), Examining pedagogical content knowledge (pp. 95-132). Dordrecht: Kluwer.

Manassero, M. A. (2013). Emociones: del olvido a la centralidad en la explicación del comportamiento. En V. Mellado, L. J. Blanco, A. B. Borrachero \& J. Cárdenas (Eds.), Las emociones en la enseñanza y el aprendizaje de las ciencias y las matemáticas (pp. 3-18). Badajoz: DEPROFE.UEX.

Mellado, V., Borrachero, A. B., Brígido, M., Melo, L. V., Dávila, M. A., Cañada, F. et al. (2014). Las emociones en la enseñanza de las ciencias. Enseñanza de las Ciencias, 32(3), 11-36. Recuperado el 10 de octubre de 2018, de: https:// ensciencias.uab.es/article/view/1478.

Olivares, J. L. \& Morales, T. C. (2013). Partir de historias académicas para comunicar, resignificar y planificar aprendizajes relevantes. Praxis Educativa, 17(2), 6471. Recuperado el 24 de febrero de 2019, de: http://www.redalyc.org/pdf/1531/ 153129924007.pdf.

Oosterheert, I. E. \& Vermunt, J. D. (2001). Individual differences in learning to teach: relating cognition, regulation an affect. Learning and Instruction, 11, 133156.

Peme-Aranega, C., Mellado, V., De Longhi, A. L., Argañaraz, M. R. \& Ruiz, C. (2008). La interacción entre las concepciones y la práctica de una profesora de Ciencias Naturales de secundaria con experiencia: Un programa longitudinal de desarrollo profesional basado en la reflexión orientada colaborativa. TED: Tecne, Episteme y Didaxis, 24, 75-98. Recuperado el 13 de marzo de 2019, de: http://reec.uvigo.es/ volumenes/volumen8/ART15_Vol8_N1.pdf.

Ritchie, S. M., Tobin, K., Hudson, P., Roth, W. M. \& Mergard, V. (2011). Reproducing successful rituals in bad times: exploring emotional interactions of a new science teacher. Science Education, 95(4), 745-765. Recuperado el 15 de agosto de 2018, de: https://onlinelibrary.wiley.com/doi/abs/10.1002/sce.20440.

Tobin, K. (2010). Reproducir y transformar la didáctica de las ciencias en un ambiente colaborativo. Enseñanza de las Ciencias, 28(3), 301-313. Recuperado el 27 de julio de 2018, de: https://www.raco.cat/index.php/Ensenanza/article/view/ 210802.

Vázquez, A. \& Manassero, M. A. (2007). En defensa de las actitudes y emociones en la educación científica (I): evidencias y argumentos generales. Revista Eureka sobre Enseñanza y Divulgación de las Ciencias, 4(2), 247-271. Recuperado el 20 de julio de 2018, de: http://www.redalyc.org/pdf/920/92040304.pdf. 